\title{
An efficient implementation of reconfigurable interpolation root- raised cosine FIR filter for software-defined radio application
}

\author{
S C PRASANNA ${ }^{1, *}$ and S P JOY VASANTHA RANI ${ }^{2}$ \\ ${ }^{1}$ Valliammai Engineering College, Anna University, Chennai 603 203, India \\ ${ }^{2}$ MIT Campus, Anna University, Chennai 600 044, India \\ e-mail: mail2scprasanna@yahoo.co.in; joy_mit@annauniv.edu
}

MS received 25 July 2015; revised 11 January 2016; accepted 24 April 2016

\begin{abstract}
This brief presents an efficient binary common subexpression elimination (BCSE)-based approach for designing reconfigurable interpolation root-raised cosine (RRC) finite-impulse-response (FIR) filter, whose coefficients change during runtime for multistandard wireless communication system called software-defined radio (SDR). Reconfiguration can be done conveniently by storing the coded coefficients in the lookup tables (LUTs), and loading the required coefficient set over the interpolation filter. In the proposed method based on 4-bit BCSE algorithm, first the number of binary common subexpressions (BCSs) formed in the coefficients is reduced. Hence, multiplexers, shifters, and adders in the multiplier structure are reduced, which results in the improvement of operating frequency. The number of addition operations is further reduced using programmable adders and an efficient polyphase interpolation structure is implemented to reduce the hardware cost. The proposed design has $49.5 \%$ less area-delay product and $28.6 \%$ improved frequency of operation when compared to a 2-bit BCSE-based technique reported earlier when implemented on Xilinx field-programmable gate array (FPGA) device XC2VP4FF672-6. Similarly, the proposed design supports $93.14 \mathrm{MHz}$ operating frequency, which is $59.2 \%$ and $74.2 \%$ greater when compared to 2-bit BCSE- and 3-bit BCSE-based approach when implemented on XC2V3000FF1152-4. The proposed structure also shows improved performance in terms of speed and area when compared to distributed arithmetic (DA)-based and multiply-accumulate (MAC)-based approaches.
\end{abstract}

Keywords. BCSE algorithm; multistandard wireless communication; reconfigurable interpolation filter; rootraised cosine filter; software-defined radio.

\section{Introduction}

In today's scenario, to meet the ever-increasing demands for the wide range of wireless services, there is a tremendous development in wireless communication technology. The concept of software-defined radio (SDR) was first envisaged by Mitola [1] in the mid-1990s, to meet the increasing demands in this field, with the aim of designing reconfigurable radio architecture that should support all cellular or noncellular standards [2]. The goal is to design an SDR by configuring the programmable hardware with the appropriate software, over-the-air depending upon the need and the radio environment, to support current and evolving new different wireless standards. This requires that the entire signal processing tasks, such as interpolation/ decimation, channel selection, equalization, and sample rate conversion in the transceiver of SDR, should be done in digital domain. This in turn requires much technological advancements in the RF frontend [3-8]. A major challenge

*For correspondence faced by the telecommunication industry today is the design of most computationally intensive part of SDR, the sample rate converter, which requires a bank of FIR filters to support different standards, with low power, flexibility, and high speed of operation. The concept of SDR can be made feasible by using a programmable logic device known as FPGA, which allows dynamic partial reconfiguration and speeds up the reconfiguration process and the power consumed by this device is also less $[9,10]$.

Several researchers have contributed toward designing a low-power, low-area, and low-complexity reconfigurable FIR filter for data rate conversion in SDR system. Chandran et al [11] presented the multiply-accumulate (MAC)-based approach to optimize the power utilization, in the implementation of reconfigurable RRC filter. It is done by optimizing the filter length based on the power of in-band and out-of-band received signal. But the MAC approach would not provide high speed of operation. Chen and Chiueh [12] proposed digit-based reconfigurable FIR filter architecture, in which it is depicted that canonic signed digit (CSD)based method serves better than the MAC approach. In the 
multiplier-less distributed arithmetic (DA) FIR filter design approach [13], various concepts such as time-multiplexed look-up tables (LUTs), offset binary coding (OBC), power gating, and memory partitioning are incorporated for reducing memory size, leakage power, and to allow reconfiguration and ultimately high throughput is achieved. However, further reduction in leakage power can be achieved only at the cost of lower throughput.

Lee et al [14] have proposed an architecture for implementing low-power reconfigurable FIR filter, in which switching activity is reduced by turning off the multiplier, based on the amplitude of the input and the coefficients. However, performance of this structure depends upon the minimum value (window size) of the amplitude of input/coefficient chosen, to turn off the multiplier. Hence, power reduction is achieved with smaller area overhead and performance degradation. The area and power optimization is achieved in realizing FIR filter, by using nonuniform quantization for bit-width optimization. It is also proposed by Hsiao et al [15] to use direct form architecture rather than transposed form to reduce the number of D flip-flops used for delay elements, and hence the efficient area and power utilization is done. However, this structure provides low speed of operation when compared to earlier designs, making it unsuitable for SDR application. A low-complexity architecture based on BCSE algorithm has been proposed by Mahesh and Vinod [16], Sagadevan et al [17], and Sandhiya et al [18]. The architecture realized using this algorithm consumes less area and power than those of CSD-CSE method using a common constant/programmable shift-and-add block. However, constant shift used in multiplier unit of FIR filter involves the use of redundant adder block as described by Mahesh and Vinod [16]. This additional hardware consumes more area and power.

Hatai et al [19] have also proposed 2-bit BCSE algorithm-based architecture for reconfigurable multistandard DUC, in which optimization in area, power, and speed is achieved by reducing multiplications per input sample (MPIS) and by reducing additions per input sample (APIS). However, the number of multiplexers needed and hence the adders needed in the multiplier block increases with increase in coefficient width. This in turn results in increased area, power, and reduced speed of operation. To overcome this issue, it is proposed to use 4-bit BCSE algorithm, to reduce the number of multiplexers, and hence number of partial products and the adders, to speed up the multiplication operation. In addition to that the number of addition operations is further reduced based on the operand values using programmable adders. Programmable multiplexers and programmable shifters make the reconfiguration of FIR interpolation filter possible.

The organization of this paper is as follows. The complexity in realizing reconfigurable interpolation RRC FIR filter and the method proposed for addressing it is explained in section 2. The architectural details of proposed reconfigurable interpolation filter for multistandard wireless communication is described in section 3. In section 4, implementation results, and discussion on the comparison of proposed design with the earlier reported results is shown. Finally, the proposed work is concluded in section 5 .

\section{Method proposed to address the issues in the design of reconfigurable interpolation $\mathrm{RRC}$ FIR filter for multistandard wireless communication}

For the purpose of comparison the three standards considered in this design are universal mobile telecommunication system (UMTS), wideband code division multiple access (WCDMA), and digital video broadcasting (DVB) as considered in [19]. With reference to the specifications [19] given in table 1, the filter coefficients of RRC FIR interpolation filter are determined for different interpolation factors, roll-off rate, and filter length (number of taps), using MATLAB.

The efficient implementation of this design requires several issues to be addressed, to make this structure suitable for SDR application. As per the discussion made in the introduction, SDR application claims high-speed operation [19] as well as less area and less complex structure to perform switch over from one standard to another over-theair and less power consumption to support mobile application [16]. The issues in designing the reconfigurable RRC interpolation filter, and method proposed to solve these issues are discussed next.

\subsection{First issue: complexity in multiplier realization}

The mathematical representation of FIR filter is represented by the equation as shown.

$$
y(n)=\sum_{k=0}^{N-1} h(k) x(n-k)
$$

where $y(n)$ is the output, $x(n)$ is the input, $h(n)$ is the coefficient, and $N$ is the number of taps of the filter. This representation shows that one of the major issues or complexities lies in the realization of FIR filter itself. The complexity in implementing FIR filter is dominated by the complexity in the implementation of multipliers. In

Table 1. Filter design specification for three standards.

\begin{tabular}{lccc}
\hline$\alpha=0.22$ & & & \\
$\alpha=0.35$ & WCDMA & UMTS & DVB \\
\hline Interp. factor & 4 & 8 & 6 \\
Sym. rate (MSPS) & 1.25 & 3.84 & 27.5 \\
Samp. freq. (MHz) & 5 & 30.72 & 165 \\
Taps & 25 & 49 & 37 \\
\hline
\end{tabular}


performing multiplication operation, the number of partial products generated increases with the increase in width of filter input and filter coefficient. This in turn increases the number of adder units and logic levels needed and hence logic depth of the structure, which consequently decreases the speed of operation of filter structure, making it unsuitable for SDR application.

The following three structures are used in the proposed method for addressing this issue when compared to earlier design [19]. Among these structures, structure 1 is based on the algorithm used in the proposed design. This algorithm plays a vital role in speed improvement by reducing the number of partial products, and thus the adders needed for multiplication. Similarly, structure 2 and structure 3 aids for further improvement in speed of multiplication operation by bypassing unnecessary addition operation. This is done by using programmable adders in structure 2 and programmable multiplexers and shifters in structure 3 .

Structure 1 Major factor for speed improvement in multiplication is achieved through this structure, by using the following specified algorithm, which results in the reduced generation of partial products and thus requires reduced number of adders. Among several techniques proposed earlier, the BCSE method is the suitable choice for implementing an efficient constant multiplier in which the number of addition operation is significantly reduced. This reduction percentage again depends upon the proper choice of length of BCS. Usually, filter coefficients are fractional and lesser in magnitude, and it requires more number of bits to represent them precisely. This requirement imposes that the proper length of BCS for the proposed architecture as 4-bit BCS, in which 2-bit, 3-bit, and 4-bit BCSs are shared among the coefficients and results in significant reduction in total number of addition operation and significant improvement in speed of operation when compared to 2-bit BCSE [19] algorithm.

\section{- 4-bit BCSE algorithm}

In BCSE algorithm, an $n$-bit binary number can form $2^{n}$ $-(n+1)$ BCS among themselves. For example, a 4-bit binary representation can form 11 BCSs, such as $\left[\begin{array}{llll}0 & 0 & 1 & 1\end{array}\right]$, [0 $\left.\begin{array}{llll}0 & 0 & 1\end{array}\right],\left[\begin{array}{llll}0 & 1 & 1 & 0\end{array}\right],\left[\begin{array}{llll}0 & 1 & 1 & 1\end{array}\right],\left[\begin{array}{llll}1 & 0 & 0 & 1\end{array}\right],\left[\begin{array}{llll}1 & 0 & 1 & 0\end{array}\right],\left[\begin{array}{llll}1 & 0 & 1 & 1\end{array}\right],\left[\begin{array}{ll}1 \\ 1\end{array}\right.$ $\left.\begin{array}{lll}1 & 0 & 0\end{array}\right],\left[\begin{array}{llll}1 & 1 & 0 & 1\end{array}\right],\left[\begin{array}{llll}1 & 1 & 1 & 0\end{array}\right]$, and [ $\left[\begin{array}{llll}1 & 1 & 1 & 1\end{array}\right]$. In the proposed 4-bit BCSE algorithm, the number of BCS is reduced from 11 to 7 (from $2^{n}-(n+1)$ to $2^{(n-1)}-1$ ) BCSs, in which the maximum size of BCS is 4-bit, and the other two sizes of BCS would be 2-bit and 3-bit, as explained next.

Here the three 4-bit BCSs [ [ $\left.\begin{array}{llll}0 & 0 & 1 & 1\end{array}\right],\left[\begin{array}{llll}0 & 1 & 1 & 0\end{array}\right]$, and [ $\left[\begin{array}{lll}1 & 1 & 0\end{array}\right.$ 0] are realized using single 2-bit BCS [1 1]. Similarly, the

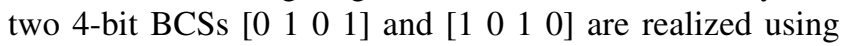
single 3-bit BCS [ $\left.\begin{array}{lll}1 & 0 & 1\end{array}\right]$, and finally the two 4-bit BCSs [0 1 $\left.\begin{array}{lll}1 & 1\end{array}\right]$ and $\left[\begin{array}{llll}1 & 1 & 1 & 0\end{array}\right]$ are realized using single 3-bit BCS [ $\begin{array}{lll}1 & 1\end{array}$ 1]. This reduction is made possible by using coded coefficients (explained in next section) and programmable shifters in the proposed structure.
Hence, the following is the list of seven 4-bit BCSs and its corresponding expression, where $x$ is the input signal.

$$
\begin{gathered}
{[11]=b c s 1=x+2^{-1} x} \\
{[101]=b c s 2=x+2^{-2} x} \\
{[111]=b c s 3=x+2^{-1} x+2^{-2} x} \\
{[1001]=b c s 4=x+2^{-3} x} \\
{[1011]=b c s 5=x+2^{-2} x+2^{-3} x} \\
{[1101]=b c s 6=x+2^{-1} x+2^{-3} x} \\
{[1111]=b c s 7=x+2^{-1} x+2^{-2} x+2^{-3} x}
\end{gathered}
$$

From Eq. (2) it can be noted that the direct implementation of BCS generation using these equations requires 12 adders. The observation of these equations depicts that, bcs $1=x+2^{-1} x$ is the common expression among four BCSs such as bcs3, bcs5, bcs6, and bcs7. Thus, sharing of bcs1 among these four BCSs, results in the following expressions for bcs3, bcs5, bcs6, and bcs7.

$$
\begin{gathered}
b c s 3=b c s 1+2^{-2} x \\
b c s 5=x+2^{-2} b c s 1 \\
b c s 6=b c s 1+2^{-3} x \\
b c s 7=b c s 1+2^{-2} b c s 1 .
\end{gathered}
$$

Therefore, the realization of BCS generation circuitry by employing the relation shown in Eqs. (3a)-(3d) and in Eqs. (2a), (2b), and (2d) results in the reduction of adders from 12 to 7 .

The number of adders required for generating BCS in 2-bit BCSE algorithm and in 3-bit BCSE algorithm is only 1 and 3, respectively. But the number of multiplexers for selecting BCS, shifters, and adders needed for implementing multipliers in reconfigurable RRC FIR interpolation filter would be more per multiplication than that needed in proposed 4-bit BCSE algorithm. For example, the 2-bit BCSE algorithm requires additional four multiplexers, four shifters, and four adders per multiplication when compared to the proposed 4-bit BCSE algorithm. This feature also makes the propagation delay of $16: 1$ multiplexers used in the proposed design becomes ignorable. Table 2 shows the comparison of number adders used between proposed method and 2-bit BCSE method for a 25-tap filter.

This comparison shows that about 54\% reduction is achieved in the addition operation in the proposed method. Therefore, this would result in reduced switching activity and hence reduced power consumption and also speeds up the computation. 
Table 2. Comparison of adders between [19] and proposed method for 25-tap filter.

\begin{tabular}{lcccr}
\hline & \multicolumn{3}{c}{ No. of adders } \\
\cline { 2 - 5 } & $\begin{array}{c}\text { BCS } \\
\text { generator }\end{array}$ & $\begin{array}{c}\text { Per multiplication for adding partial } \\
\text { products }\end{array}$ & $\begin{array}{c}\text { For adding partial products of 25-tap } \\
\text { filter }\end{array}$ & $\begin{array}{c}\text { Total no. of } \\
\text { adders }\end{array}$ \\
\hline 2-bit BCSE [19] & 1 & 7 & 175 & 176 \\
Proposed 4-bit & 7 & 3 & 75 & 82 \\
BCSE & & & & \\
\hline
\end{tabular}

Structure 2 This structure also helps in improving speed by further reducing the number of addition operations, when possible, based on the operand (partial product) values. This is accomplished by using programmable adders, which permits the execution of addition operation only when both the partial products to be added are non-zero. When any one of the partial product or both the partial products at its input are zero, then the sum will be immediately made available at its output through multiplexer, thus eliminating unnecessary addition operation and hence reducing switching activity as well.

For example, for the RRC FIR interpolation filter that supports UMTS standard with filter taps of 49, interpolation factor of 8 , and roll-off factor of 0.22 , the number of addition operation performed using the proposed method is 104, whereas earlier design using 2-bit BCS is 343, which uses fixed adder structure. This shows that the proposed method provides almost about $70 \%$ reduction in addition operation. Even if the programmable adder is used in 2-bit BCS, the number of addition operation that would be performed is 196, which is still about twice the adders used in the proposed method.

Structure 3 Again structure 3 also aids in reducing addition operation, when possible, based on the maximum number of BCS found in a coefficient. The programmable multiplexers and programmable shifters are used to perform this task. The number of multiplexers needed for selecting BCS is equivalent to maximum number of BCS found in the coefficient. Consequently, number of shifters and hence the number of adders used in evaluating the product can also be reduced based on the maximum number of BCS found in the coefficient, whereas these are fixed in [19].

\subsection{Second issue: complexity in interpolation filter realization}

Another complexity lies in the realization of reconfigurable interpolation operation. The complexity is that the direct implementation of N-tap interpolation RRC FIR filter requires $\mathrm{N}$-multipliers. This complexity grows with the reconfigurable interpolation RRC FIR filter, as it has to support different and larger number of taps upon switch over from one wireless standard to another standard. The solution to this complexity is addressed, using polyphase interpolation structure as explained next.

Solution The interpolation RRC FIR filter is implemented using efficient polyphase interpolation structure. The polyphase decomposition of an N-tap FIR filter with the system function shown in Eq. 4 is described as follows:

$$
H(z)=\sum_{n=0}^{N-1} h[n] z^{-n}
$$

The polyphase decomposition of Eq. 4, for an interpolation factor of $L$, is obtained by expanding and transforming this into the form

$$
H(z)=\sum_{i=0}^{L-1} H_{i}\left(z^{L}\right) \cdot z^{-i}
$$

where

$$
H_{i}(z)=\sum_{n=0}^{\left(\frac{N}{L}\right)-1} h[n L+i] z^{-n} \quad \text { and } \quad i=0,1,2, \ldots, L-1
$$

Equation (5) shows that the system function $H(z)$ is decomposed into L-subfilters, and each $H_{i}(z)$ would be treated as an independent subfilter's system function with $N / L$ taps.

Therefore, in polyphase interpolation structure, an N-tap FIR interpolation filter with interpolation factor of $L$ can be realized using L-subfilters of each with $N / L$ taps and $L-1$ structural adders. The realization of each subfilter requires $N / L$ multipliers and structural adders. Realization of single subfilter is made sufficient for the implementation of the complete filter, by sequentially loading the subfilter L-times with appropriate coefficients and accumulating its output. Thus, this reduces the complexity of FIR interpolation filter realization from $\mathrm{N}$-tap to $(N / L)$-tap.

\subsection{Third issue: complexity in reconfiguration}

Again the complexity lies in the realization of reconfigurable RRC FIR interpolation filter to support multistandard wireless communication. This realization requires filters with different taps and different interpolation factors 
to be realized. The implementation of such structure requires $\mathrm{L} 1+\mathrm{L} 2+\mathrm{L} 3+\cdots$ subfilters and $\left[\frac{N 1}{L 1}\right]+\left[\frac{N 2}{L 2}\right]+$ $\left[\frac{N 3}{L 3}\right]+\cdots$ multipliers and structural adders, for filters with taps $\mathrm{N} 1, \mathrm{~N} 2, \mathrm{~N} 3, \ldots$ and interpolation factors $\mathrm{L} 1, \mathrm{~L} 2, \mathrm{~L} 3, \ldots$, respectively.

Solution Solution to multistandard support is based on the fact that, at any instant, only one required configuration/standard is needed to be realized on the reconfigurable multistandard interpolation filter. So, in the proposed method, a single subfilter would be shared among different filters by dynamically loading it with the required coded coefficients for filters with, say, $\left[\frac{N 1}{L 1}\right]=\left[\frac{N 2}{L 2}\right]=\left[\frac{N 3}{L 3}\right]=a$, where $a$ is a constant. This reduces the realization of number of subfilters and hence the adders, at the cost of maintaining this ratio equivalent to constant $a$. In addition, the coefficient symmetry property of FIR filter is also exploited in this method, which halves the memory size needed for storing the coefficients.

\section{Proposed architecture}

The general block diagram of the proposed architecture for reconfigurable RRC FIR interpolation filter for multistandard wireless communication is shown in figure 1. This diagram shows that the reconfigurable RRC FIR interpolation filter is loaded with any of six coefficient sets to support any of WCDMA, UMTS, or DVB wireless standard. The appropriate coefficients are fetched from coefficient storage unit called LUTs using INTERP_SEL and FILTER_SEL signals, which are used for selecting the desired interpolation factor (wireless standard) and roll-off factor, respectively, and are loaded over reconfigurable RRC FIR interpolation filter. The signal DATA_IN is the filter input to be interpolated, and CLK defines the

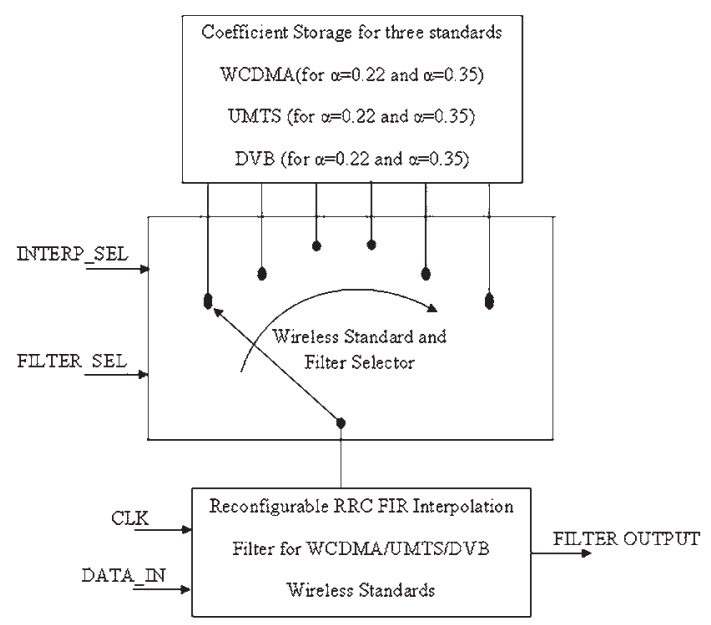

Figure 1. General block diagram for proposed method. sampling frequency of the input signal DATA_IN, and finally FILTER_OUTPUT is the pulse-shaped interpolated output signal.

The proposed architecture for reconfigurable RRC FIR interpolation filter is shown in figure 2. For the purpose of comparison, the proposed structure is designed for 25-, 37-, and 49-tap filters with interpolation factors 4, 6, and 8, respectively, and each with two different roll-off factors 0.22 and 0.35 . The major blocks are clock and data generator, BCS generator, coded coefficient steering unit, partial product generation and programmable adder unit, subfilter-accumulation unit, and interpolation filter-accumulation unit.

The sequence of operation of this proposed structure is briefed as follows:

- Six set of coefficients are determined in its 16-bit 2's complement form with reference to the specification given in table 1. Coded form (BCSs and its corresponding shift) of these coefficients are stored in six different LUTs.

- Clock generator generates the DATA_CLK (sampling frequency of DATA_IN) signal from CLK signal by dividing it by the interpolation factor.

- BCS generator generates BCSs in synchronization with DATA_CLK signal.

- Address generator generates an address based on the interpolation factor in synchronization with CLK signal.

- Coded coefficients are fetched from LUTs using this generated address and roll-off factor, which functions as the BCS selection input signal for BCS selector unit and shift select input signal for programmable shifter unit.

- The selected BCS and/or $x \_$in is shifted appropriately, resulting the generation of partial products.

- These partial products are accumulated using programmable adders, to get the product.

- These products are accumulated using structural adders in subfilter-accumulation unit.

- Finally, L (interpolation factor) subfilter outputs are combined to generate pulse-shaped interpolated filter output.

The detailed description of each block of the proposed architecture is presented in the following text.

\subsection{Clock and data generator}

The clock generator is used for generating the required sampling frequency signal, based on the interpolation rate. The master clock signal (CLK) is the high-frequency input signal to the clock generator as shown in figure 2. To perform interpolation, the coefficients and output unit of the filter must be processed at a rate equal to interpolation 


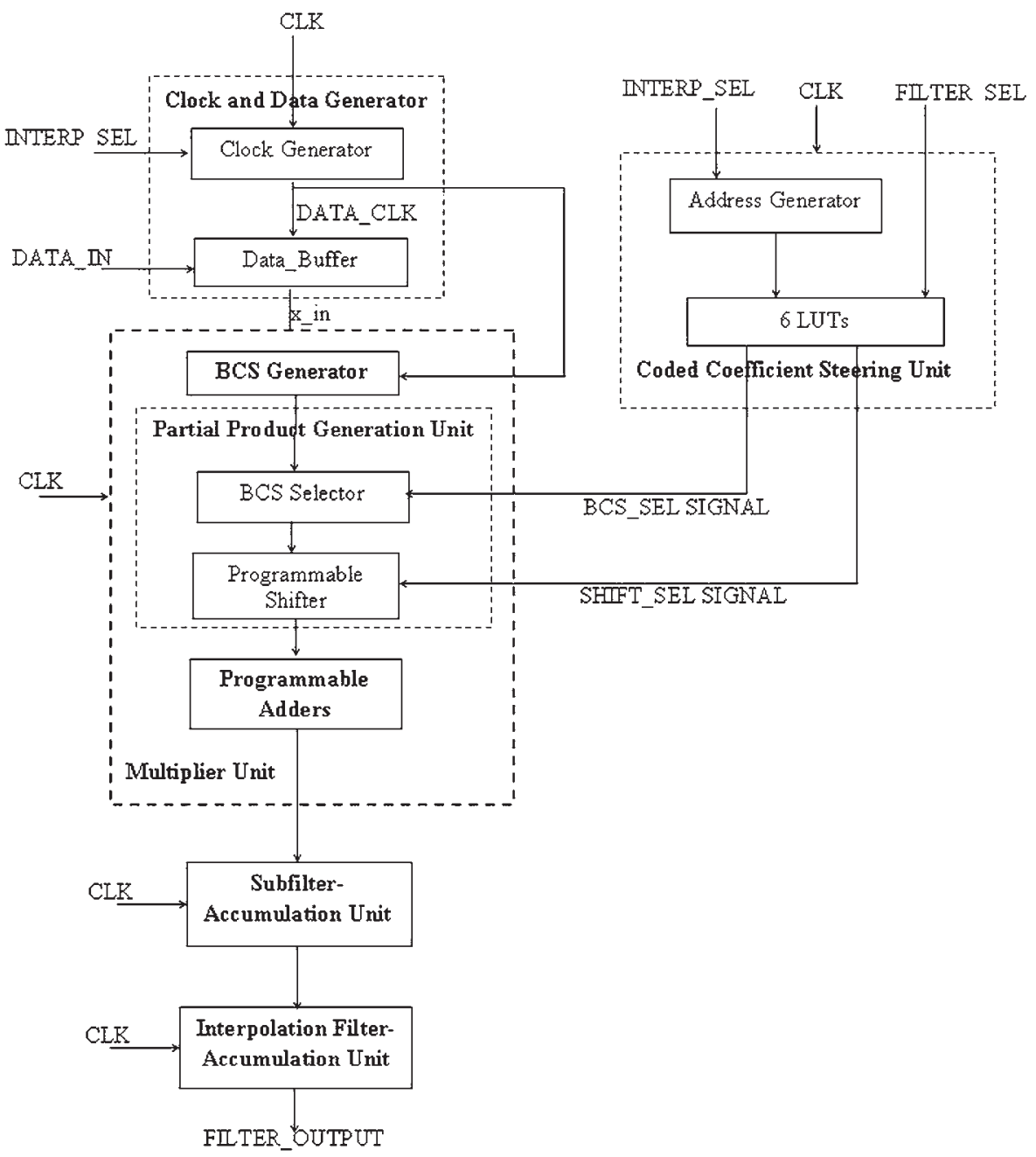

Figure 2. Proposed architecture.

factor times the sampling frequency of data input to the proposed structure. The coefficients and output unit of the filter is processed using master clock. The data input sampling frequency signal for three different interpolation factors 4,6 , and 8 is generated by dividing the master clock (CLK) by 4 (CLKby4), 6 (CLKby6), and 8 (CLKby8), respectively. This unit generates only the desired data input sampling frequency signal (DATA_CLK) based on the interpolation factor, which serves as the operating frequency for the data generator (or data buffer) unit and BCS generator. All other modules in the proposed architecture are operated at master clock frequency to achieve required interpolation.

Data generator is simply a register/buffer that receives the data input of the reconfigurable interpolation filter architecture and outputs it to the BCS generator unit (x-in), at a rate CLKby 4 or CLKby6 or CLKby8, which is provided by the clock generator.

\subsection{BCS generator}

It is already explained that the 4-bit BCSE algorithm is the suitable choice for the design example considered here. All the possible BCSs of this algorithm and its expressions are discussed earlier in this paper. The structure used for realizing these seven BCSs are shown in figure 3. The structure shows that bcs1, bcs2, and bcs 4 are generated in the first logic level and the remaining bcs3, bcs5, bcs6, and bcs 7 are generated in second logic level using only seven adders. The total propagation time of this proposed unit is, $t_{\text {prop_bcs_gen }}=2 * t_{\text {prop_adder. The propagation time of this }}$ unit for 2-bit BCSE is [19], $t_{\text {prop_bcs_gen }}=1 * t_{\text {prop_adder, }}$ as it requires only one logic level. But the total propagation time of proposed structure is reduced compared to [19]. This is because the BCS is generated only once for given input and shared among all the multipliers. As explained in the previous section, the number of multiplexers, shifters, and 


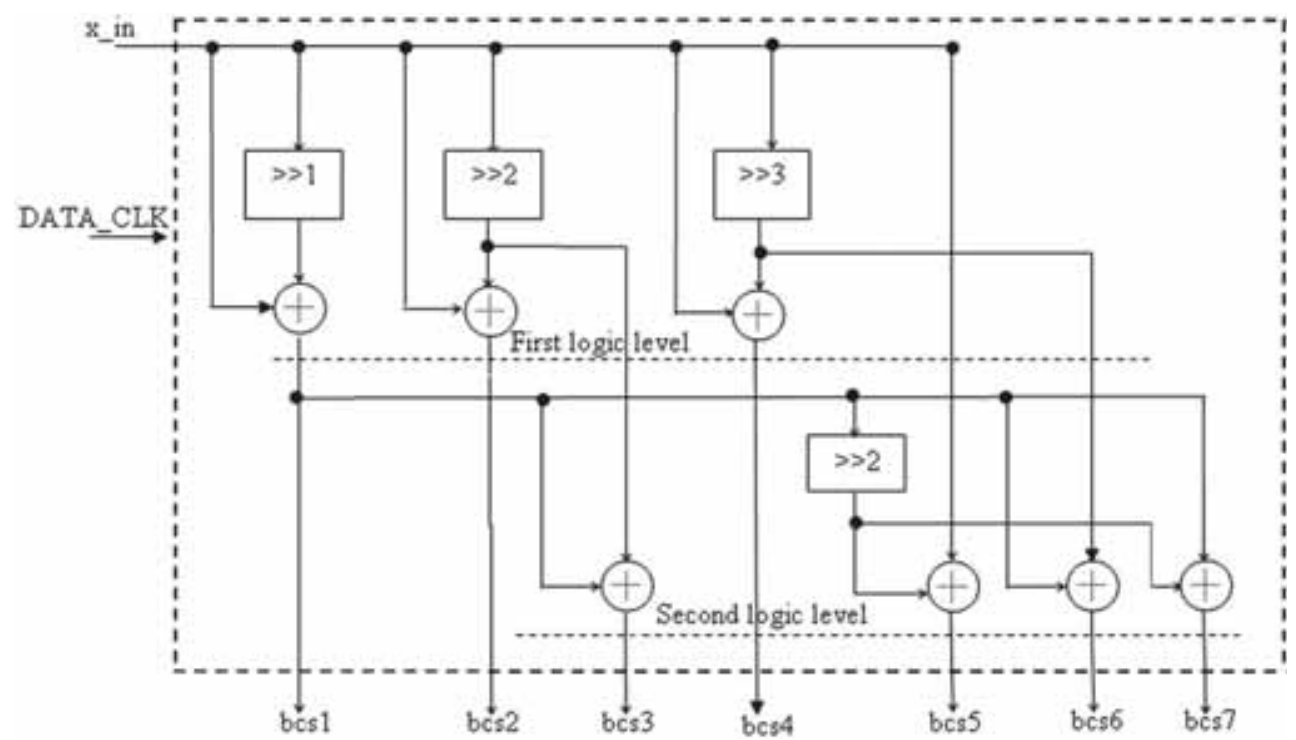

Figure 3. Two-level BCS generator.

adders needed per multiplier is more in 2-bit BCSE; consequently, there is a significant increase in the time and area for the overall structure, which also grows with increased number of taps.

\subsection{Coded coefficient steering unit}

This unit consists of address generator, two LUTs (one for roll-off factor 0.22 and another for 0.35 ) per standard for storing the coded coefficients, and multiplexers for selecting the desired coded coefficients based on the roll-off factor and interpolation factor as shown in figure 4. The concept of coefficient coding, number of multiplexers for BCS selection, address generation logic based on interpolation rate, and coefficients utilized in subfilter structure for three interpolation factors 4,6 , and 8 are described next.

Coefficient coding The concept of coefficient coding is explained with the following example. Consider the coefficient $h$ in its 2's complement binary form,

$$
h=[0 \underline{00101010011110}] .
$$

The BCSs found in this coefficient according to our defi-

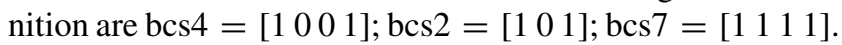
Based on the position of these BCSs, they should be shifted as follows. The bcs4, bcs 2 , and bcs 7 should be shifted toward right by 1-, 6-, and 11-bit positions, respectively. Therefore, the bcs and its corresponding shift value is stored in each location of the LUT, instead of the coefficient itself. This method permits the use of programmable multiplexers and shifters that simplifies reconfiguration process and also improves speed of operation. For the coefficient mentioned, the coded value stored in the LUT is $h=[\{1,4\},\{6,2\},\{11,7\},\{0,0\}]$. Here in each \{\} , first value represents the shift needed and the second value represents the BCS needed in the process of generation of partial product.

In the considered design example for the proposed method, the maximum number of 4-bit BCSs found in the coefficient sets is 4 . Therefore, using 8-bits per BCS (4-bits to represent/select the BCS and 4-bits to specify the required shift for that selected BCS), each coefficient is coded using 32-bits. In spite of this area overhead, the proposed method provides increased frequency of operation as is needed for SDR application. These coded coefficients, which are the outputs of the coded coefficient steering unit, are used for selecting the desired BCS and for performing the desired shift operation over selected BCS.

The proposed method employs only horizontal BCS (not vertical BCS), so coefficient symmetry property of FIR filter, $h(n-1-n)=h(n)$ is employed in storing the coded coefficients. Using this symmetry property, only $50 \%$ of the coded coefficients, that is, two sets of 13,19 , and 25 coefficients are stored instead of two sets of 25,37 , and 49 coefficients to support three different interpolation factors 4,6 , and 8 , respectively. One set for roll-off factor 0.22 and another for roll-off factor 0.35 . This implies that this property greatly reduces the number of locations needed from 222 to 114, that is almost about $50 \%$ reduction in usage of LUT area/size is achieved.

Address generation logic The address generator generates the desired address sequence based on the interpolation factor to fetch the required coefficients from the LUT in synchronization with master clock frequency (CLK). This logic is realized depending upon the realization of the proposed structure as explained below. 


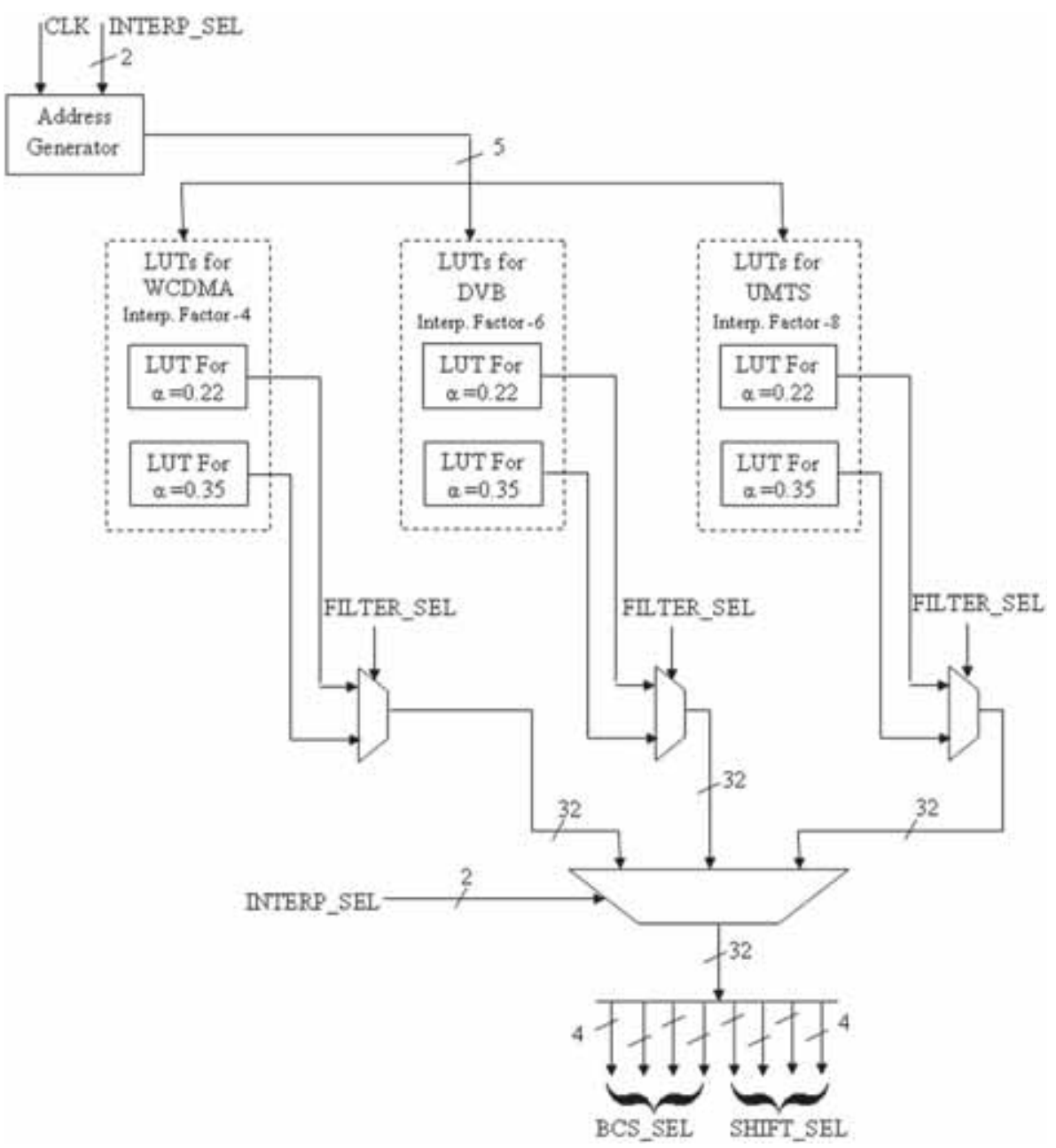

Figure 4. Coded coefficient steering unit.

The polyphase realization of proposed reconfigurable interpolation RRC FIR filters are realized using subfilters of length 7 , as $\left[\frac{25}{4}\right]=\left[\frac{37}{6}\right]=\left[\frac{49}{8}\right]=7$. This shows that the same subfilter can be dynamically loaded with the appropriate coefficients to achieve required interpolation and characteristics. The number of subfilters needed for interpolation factors 4, 6, and 8 is 4, 6, and 8, respectively. Each subfilter is simply an FIR filter; here it is realized in transposed direct form structure as shown in figure 7, which is the suitable choice for high-speed operation rather than direct form structure. The coefficients allocated for realizing the corresponding subfilters for three different interpolation factors is shown in table 3 .

Therefore, the address generation sequence is 0,4 , $8, \ldots, 24 ; 1,5, \ldots, 25 ; 2,6, \ldots, 26$; and $3,7, \ldots, 27$ for interpolation factor of 4 . In a similar manner, the required address sequence for interpolation of 6 and 8 is generated with reference to table 3 . Four-bits are needed for specifying the address of 13 coefficients for interpolation factor of 4 , whereas 5-bits are needed for specifying the address of 19 and 25 coefficients for interpolation factors of 6 and 8 , respectively. Four-bit address is represented in 5-bits by appending ' 0 ' at its MSB. Therefore, single set of 5-bit address line is used for accessing LUTs of all the standards considered here as shown in figure 4.

Finally, three 2-input multiplexers are used for selecting the coefficients based on the roll-off factor (FILTER_SEL), this halves the number coefficients passed on to the next stage. Finally, a three-input multiplexer steers only the desired coded coefficient based on the interpolation factor and is made available at the output lines of this unit.

\subsection{Partial product generation and programmable adder unit}

The internal structure of this unit is shown in figure 5. All the partial products per multiplication are generated simultaneously from DATA_IN, BCSs (bcs1, bcs2, bcs3, bcs4, bcs5, bcs6, bcs7) and coded coefficients. The coded coefficients 
Table 3. Allocation of coefficients for subfilters.

\begin{tabular}{|c|c|c|c|}
\hline & Interpolation factor -4 & Interpolation factor -6 & Interpolation factor -8 \\
\hline $\begin{array}{l}\text { Subfilter } \\
\quad 1\end{array}$ & $h(0), h(4), h(8), h(12), h(16), h(20), h(24)$ & $\begin{array}{c}h(0), h(6), h(12), h(18), h(24), h(30), \\
h(36)\end{array}$ & $\begin{array}{c}h(0), h(8), h(16), h(24), h(32), h(40), \\
h(48)\end{array}$ \\
\hline $\begin{array}{l}\text { Subfilter } \\
\quad 2\end{array}$ & $h(1), h(5), h(9), h(13), h(17), h(21), h(25)$ & $\begin{array}{c}h(1), h(7), h(13), h(19), h(25), h(31), \\
h(37)\end{array}$ & $\begin{array}{c}h(1), h(9), h(17), h(25), h(33), h(41), \\
h(49)\end{array}$ \\
\hline $\begin{array}{l}\text { Subfilter } \\
\quad 3\end{array}$ & $\begin{array}{c}h(2), h(6), h(10), h(14), h(18), h(22), \\
h(26)\end{array}$ & $\begin{array}{c}h(2), h(8), h(14), h(20), h(26), h(32), \\
h(38)\end{array}$ & $\begin{array}{c}h(2), h(10), h(18), h(26), h(34), h(42), \\
h(50)\end{array}$ \\
\hline $\begin{array}{l}\text { Subfilter } \\
\quad 4\end{array}$ & $\begin{array}{c}h(3), h(7), h(11), h(15), h(19), h(23), \\
h(27)\end{array}$ & $\begin{array}{c}h(3), h(9), h(15), h(21), h(27), h(33), \\
h(39)\end{array}$ & $\begin{array}{c}h(3), h(11), h(19), h(27), h(35), h(43), \\
h(51)\end{array}$ \\
\hline $\begin{array}{l}\text { Subfilter } \\
5\end{array}$ & & $\begin{array}{c}h(4), h(10), h(16), h(22), h(28), h(34), \\
h(40)\end{array}$ & $\begin{array}{c}h(4), h(12), h(20), h(28), h(36), h(44), \\
h(52)\end{array}$ \\
\hline $\begin{array}{l}\text { Subfilter } \\
\quad 6\end{array}$ & & $\begin{array}{c}h(5), h(11), h(17), h(23), h(29), h(35), \\
h(41)\end{array}$ & $\begin{array}{c}h(5), h(13), h(21), h(29), h(37), h(45), \\
h(53)\end{array}$ \\
\hline $\begin{array}{l}\text { Subfilter } \\
7\end{array}$ & & & $\begin{array}{c}h(6), h(14), h(22), h(30), h(38), h(46), \\
h(54)\end{array}$ \\
\hline Subfilter & & & $h(7), h(15), h(23), h(31), h(39), h(47)$ \\
\hline 8 & & & $\mathrm{~h}(55)$ \\
\hline
\end{tabular}

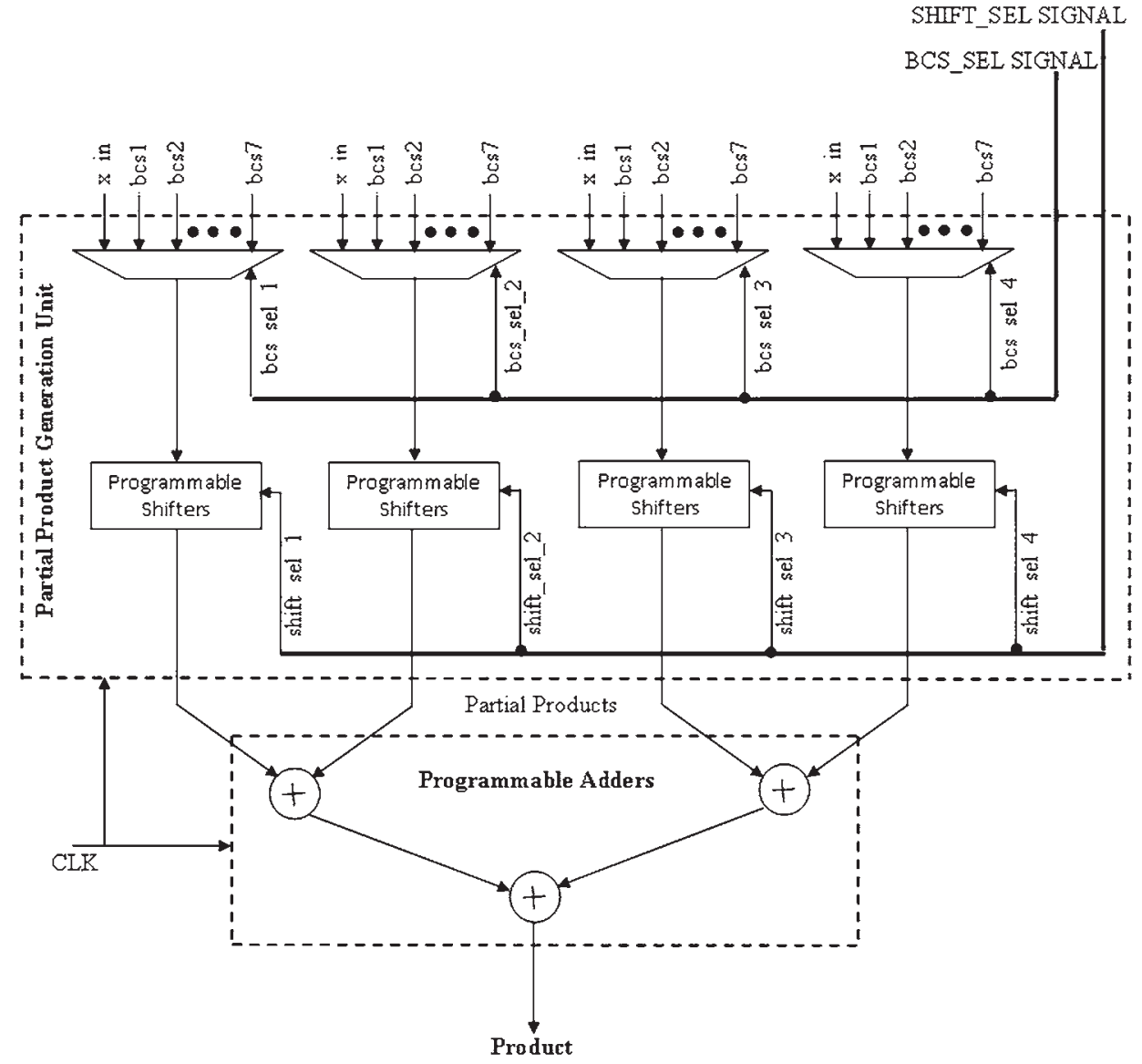

Figure 5. Multiplier unit.

provide selection line input to multiplexers for selecting the BCS and/or DATA_IN and shift select input to programmable shifters for providing the required shift and hence providing the partial products. As stated earlier, the maximum number of BCS that can be formed in the coefficient set is 4 , so four multiplexers and four shifters are used. 
These partial products are added using two-level programmable adder structure to evaluate the product as shown in figure 6. From the above discussion it is clear that the coefficients would have one, two, three, or four BCS, and hence the corresponding number of non-zero partial products would be one, two, three, or four, respectively. Now using the programmable adders in figure 6 , the number of addition operations performed is greatly reduced (as stated in the previous section), by performing addition only when both the inputs (partial products) to the adder are non-zero. Otherwise, addition operation is bypassed and the corresponding product is immediately made available at its output through multiplexer in the process of evaluation of product.

\subsection{Subfilter-accumulation unit}

Subfilter of the proposed reconfigurable multistandard interpolation filter is realized in transposed direct form structure as shown in figure 7. As mentioned earlier, each standard requires a 7-tap subfiter, so a single subfilter would be dynamically reconfigured with the required coefficients

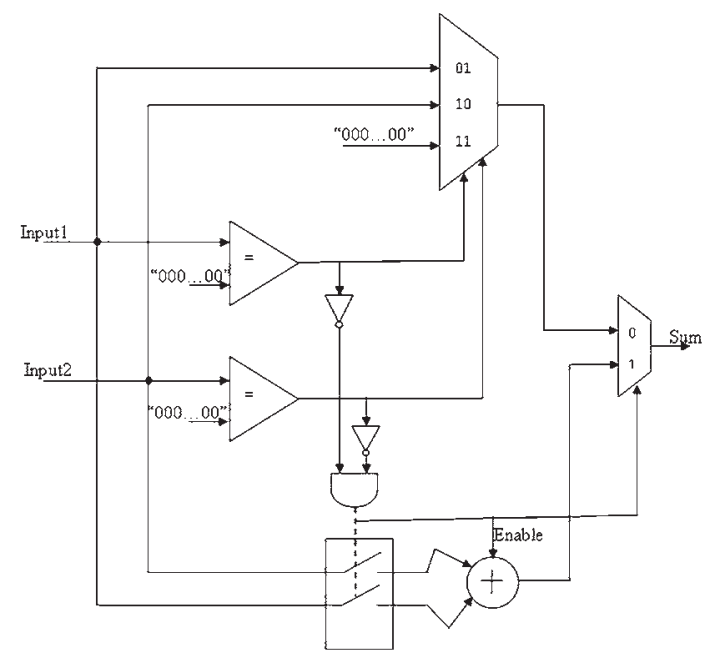

Figure 6. Programmable adder. to support the required wireless standard. Multiplication is performed using the proposed BCSE algorithm. Then these products are accumulated using accumulation unit of subfilter, which is realized using a chain of six registers and six adders.

\subsection{Filter-accumulation unit}

The output of the subfilter is accumulated again using chain of registers and adders that are operated at interpolated frequency to get the pulse-shaped interpolated output. The number of registers and the number of adders used in this accumulation unit is based on the interpolation factor. For the design example considered in the proposed architecture, the number of registers as well as adders required in the accumulation unit of reconfigurable interpolation RRC FIR filter is equivalent to 3,5 , and 7 for interpolation factors 4 , 6 , and 8 , respectively.

\section{Results and discussion}

The proposed design for reconfigurable FIR interpolation filter to support multistandard wireless communication has been implemented on XC2V3000FF1152-4 FPGA device using Xilinx ISE 9.2i EDA tool. The result of the proposed design using 16-bit input width and 16-bit coefficient width is presented in table 4 , and is compared with the structure implemented using 2-bit BCSE algorithm [19] and 3-bit BCSE algorithm (Mahesh and Vinod [16]). Comparison shows that the proposed design provides $59.2 \%$ and $74.2 \%$ improvement in speed of operation when compared to [19] and Mahesh and Vinod [16], respectively.

The proposed design is also implemented on other Xilinx FPGA devices as shown in table 5, and is synthesized using Xilinx Synthesis Tool Xilinx ISE 9.2i EDA tool. The two parameters, speed and area-delay product (ADP), are considered now for fair comparison of the proposed design with earlier designs.

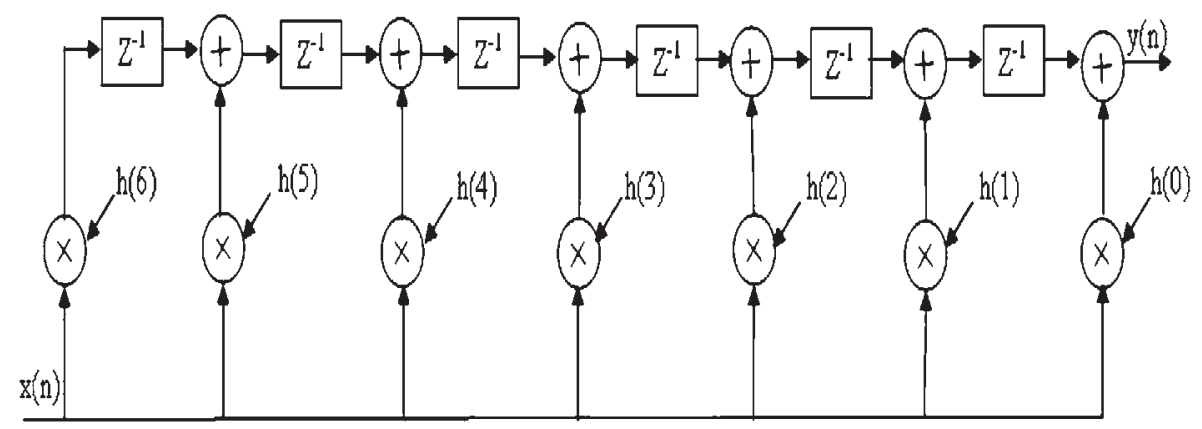

Figure 7. Transposed direct form realization of subfilter. 
Table 4. Comparison of speed with [16, 19].

\begin{tabular}{lccc}
\hline & Proposed & Hatai et al [19] & Mahesh and Vinod [16] \\
\hline Method & 4-bit BCSE & 2-bit BCSE & 3-bit BCSE \\
Word length & 16 -bit & 16-bit & 16-bit \\
Slices & 2258 & - & - \\
Delay (ns) & 10.73 & 26.31 & 41.66 \\
Freq. (MHz) & 93.14 & 38 & 24 \\
Speed improvement of proposed design (\%) & & $59.2 \%$ & $74.2 \%$ \\
\hline
\end{tabular}

Table 5. Comparison of results on different FPGA platform.

\begin{tabular}{|c|c|c|c|c|c|c|c|c|}
\hline $\begin{array}{l}\text { FPGA } \\
\text { device }\end{array}$ & References & Method & $\begin{array}{l}\text { Filter } \\
\text { length }\end{array}$ & $\begin{array}{l}\text { Delay } \\
\text { (ns) }\end{array}$ & $\begin{array}{l}\text { Max. } \\
\text { freq. } \\
(\mathrm{MHz})\end{array}$ & Slices & $\begin{array}{c}\text { Speed } \\
\text { Improvement of } \\
\text { proposed design }(\%)\end{array}$ & $\begin{array}{c}\text { ADP } \\
\text { Improvement of } \\
\text { proposed design (\%) }\end{array}$ \\
\hline \multirow[t]{3}{*}{$\mathrm{XC} 2 \mathrm{VP} 4$} & Proposed & 4-bit BCSE & $\begin{array}{c}25 / 37 / 49 \text { Tap } \\
16 \times 16\end{array}$ & 8.55 & 116.9 & 2224 & & \\
\hline & [19] & 2-bit BCSE & $\begin{array}{c}25 / 37 / 49 \text { Tap } \\
16 \times 17\end{array}$ & 11.99 & 83.4 & 3142 & 28.6 & 49.5 \\
\hline & {$[11]$} & MAC based & $\begin{array}{c}65 \text { Tap } \\
16 \times 16\end{array}$ & 16.75 & 59.7 & 9942 & 48.9 & 88.58 \\
\hline \multirow[t]{3}{*}{ XCV2000E } & Proposed & 4-bit BCSE & $\begin{array}{c}25 / 37 / 49 \text { Tap } \\
16 \times 16\end{array}$ & 18.44 & 54.2 & 2188 & & \\
\hline & [19] & 2-bit BCSE & $\begin{array}{c}25 / 37 / 49 \text { Tap } \\
16 \times 17\end{array}$ & 20.20 & 49.5 & 1788 & 8.7 & -2 \\
\hline & [20] & DA- based & $\begin{array}{c}65 \text { Tap } \\
8 \times 8\end{array}$ & 15.62 & 64 & 1061 & - & - \\
\hline
\end{tabular}

From the table it can be noticed that the synthesis results on the device XC2VP4 shows that there is an improvement in the speed as well as ADP. The propagation delay in the proposed architecture is $8.55 \mathrm{~ns}$, whereas in [19] it is 11.99 ns and in [11] $16.75 \mathrm{~ns}$, this results in the speed improvement of $28.6 \%$ and $48.9 \%$ when compared to [19] and [11], respectively. Similarly the ADP, which is one of the important figures of merit of the design, is improved by $49.5 \%$ and $88.58 \%$ when compared to [19] and [11], respectively. Comparison results on synthesizing the proposed architecture over XCV2000E with [19] shows that the propagation delay of the proposed architecture is less and provides speed improvement of $8.7 \%$ with smaller insignificant area overhead. Comparison with the architecture in [20] shows that the propagation delay for implementing $8 \times 8$ multiplier itself is $15.62 \mathrm{~ns}$, whereas the proposed design is implemented using $16 \times 16$ multiplier and it requires only $18.44 \mathrm{~ns}$, which is lesser when compared to the multiplier size used. In a similar manner, it can be justified that the number of slices utilized is actually less in the proposed design when compared with [20].

Again the proposed design is also implemented on other Xilinx FPGA device XC5VSX95TFF1136-1. Finally, for the purpose of comparison, the proposed 4-bit BCSE algorithm is implemented even in realizing smaller width $(8 \times 8)$ multiplier in a 16-tap FIR filter, on the same FPGA device. The results are tabulated in table 6 to exhibit the comparison. The comparison of proposed 16-tap filter with that in Park and Meher [21] shows that the proposed structure improves the speed by $55.08 \%$ when compared to the DA-based approach, with area overhead; this is due to the fact that multiplier size is only $8 \times 8$. But this area overhead would not be present when the width of the multiplier was increased in Park and Meher [21] equivalent to proposed example. It is also noticed that the propagation delay of the proposed reconfigurable interpolation RRC FIR filter that supports 25-, 37-, or 49-tap filters of multiplier size $16 \times 16$ is only $5.27 \mathrm{~ns}$, whereas it is $10.91 \mathrm{~ns}$ for a single 16-tap filter of multiplier size $8 \times 8$ implemented using DA-based approach (Park and Meher [21]).

Based on all the aforementioned comparisons, the proposed structure provides speed improvement in all the cases. The comparison of the proposed architecture with equal or almost similar size filters shows that there is an improvement in speed as well as ADP (over few devices) in the proposed structure. 
Table 6. Comparison of result on XC5VSX95TFF1136-1.

\begin{tabular}{lccc}
\hline & \multicolumn{2}{c}{ Proposed 4-bit BCSE } & \multicolumn{2}{c}{ Park and Meher [21] DA-based } \\
\cline { 2 - 3 } Filter length & $25 / 37 / 49$ Tap & 16 Tap & 16 Tap \\
\hline Number of slice registers & $16 \times 16$ & $8 \times 8$ & 397 \\
Number of slice LUTs & 1329 & 681 & 277 \\
Slice, number used as logic & 2970 & 599 & 126 \\
Delay (ns) & 2884 & 599 & 10.91 \\
Freq. (MHz) & 5.27 & 4.9 & 91 \\
Speed improvement of proposed design (\%) & 189.64 & 204.06 & 55.08 \\
\hline
\end{tabular}

\section{Conclusion}

The problems in designing the reconfigurable interpolation filter for multistandard wireless communication were discussed and solution to those problems is proposed and implemented on various FPGA devices. The proposed method aims at reducing the adders, and addition operation to improve speed, reduce switching activity as well as area occupied to make the structure suitable for SDR application. Comparison of results of proposed 4-bit BCSE-based reconfigurable filter with other similar structure implemented using 2-bit BCSE algorithm over the FPGA device XC2V3000FF1152-4 provides 59.2\% speed improvement, and over the FPGA device XC2VP4FF672-6 provides $28.6 \%$ improvement in frequency of operation and $49.5 \%$ improvement in ADP. Area optimization would also result in reduced power consumption. All the other comparisons of results with earlier design discussed in the previous section reveal that the proposed structure is optimized in terms of speed and area, making it a suitable reconfigurable interpolation filter for SDR application.

\section{References}

[1] Joe Mitola 1995 The software radio architecture. IEEE Commun. Mag. 33(5): 26-38

[2] Francois Rivet, Yann Deval, Jean-Baptiste Begueret, Dominique Dallet, Philippe Cathelin and Didier Belot 2008 A disruptive receiver architecture dedicated to software-defined radio. IEEE Trans. Circuits Syst-II: Exp. Briefs 55(4): 344-348

[3] Asad A Abidi 2007 The path to the software-defined radio receiver. IEEE J. Solid-State Circuits 42(5): 954-966

[4] Cristina de la Morena-Álvarez-Palencia, David RodríguezAparicio and Mateo Burgos-García 2010 Software-defined radio technologies for emergency and professional communications. IEEE International Carnahan Conference on Security Technology, San Jose, CA, 357-363

[5] Martin P, Praveen R, Min L, Antoine D, Liesbet Van der P and Francky C 2010 Future software-defined radio platforms and mapping flows. IEEE Signal Process. Mag. 27(2): 22-33
[6] Sabbir A Osmany, Frank Herzel and J Christoph Schett 2010 An integrated $0.6-4.6 \mathrm{GHz}, 5-7 \mathrm{GHz}, 10-14 \mathrm{GHz}$, and 20-28 GHz frequency synthesizer for software-defined radio applications. IEEE J. Solid-State Circuits 45(9): 1657-1668

[7] Tore Ulversoy 2010 Software defined radio: Challenges and opportunities. IEEE Commun. Surveys Tutorials 12(4): 531-550

[8] Walter H W Tuttlebee 1999 Software-defined radio: Facets of a developing technology. IEEE Personal Commun. 6(2): 38-44

[9] Rahul K, Joshi R C and Kota Solomon Raju 2009 A FPGA partial reconfiguration design approach for RASIP SDR. IEEE India Conference

[10] Wang L and Wu F-Y 2009 Dynamic partial reconfiguration in FPGAs. IEEE Third International Symposium on Intelligent Information Technology Application. 445-448

[11] Chandran J, Kaluri R, Singh J, Owall V and Velijanovski R 2004 Xilinx Virtex II Pro implementation of a reconfigurable UMTS digital channel filter. In: Proceedings of IEEE Workshop Electronic Design, Test, and Application 77-82

[12] Chen K -H and Chieueh T -D 2006 A low-power digit-based reconfigurable FIR filter. IEEE Trans. Circuits Syst. II, Exp. Briefs 53(8): 617-621

[13] Sheikh F, Miller M, Richards B, Markovic D and Nikolic B 2010 A 1-190 MSample/s 8-64 tap energy-efficient reconfigurable FIR filter for multi-mode wireless communication. In: Proceedings of IEEE Symposium on VLSI Circuits 207-208

[14] Lee S -J, Choi J -W, Kim S W and Park J 2011 A reconfigurable FIR filter architecture to trade off filter performance for dynamic power consumption. IEEE Trans. Very Large Scale Integr. (VLSI) Syst. 19(12): 2221-2228

[15] Hsiao S -F, Zhang Jian J -H and Chen M -C 2013 Low-cost FIR filter designs based on faithfully rounded truncated multiple constant multiplication/accumulation. IEEE Trans. Circuits Syst. II, Exp. Brief 60(5): 287-291

[16] Mahesh R and Vinod A P 2010 New reconfigurable architectures for implementing FIR filters with low complexity. IEEE Trans. Comput.-Aided Des. Integr. Circuits Syst. 29(2): 275-288

[17] Sagadevan K, Babu V and Arivumani Samson S 2012 Novel reconfigurable architecture with low complexity FIR filter. In: Proceedings of IEEE International Conference on PRIME 145-151

[18] Sandhiya V, Karthick S and Valarmathy M 2014 A survey of new reconfigurable architectures for implementing FIR filters 
with low complexity. In: Proceedings of IEEE International Conference on Computer Communication and Informatics: $1-9$

[19] Indranil Hatai, Indrajit Chakrabarti and Swapna Banerjee 2015 An efficient VLSI architecture of a reconfigurable pulse-shaping FIR interpolation filter for multistandard DUC. IEEE Trans. VLSI Syst. 23(6): 1150-1154
[20] Meher P K, Chandrasekaran S and Amira A 2008 FPGA realization of FIR filters by efficient and flexible systolization using distributed arithmetic. IEEE Trans. Signal Process. 56(7): 3009-3017

[21] Park S Y and Kumar Meher P 2014 Efficient FPGA and ASIC realizations of a DA-based reconfigurable FIR digital filter. IEEE Trans. Circuits Syst. II, Exp. Briefs 61(7): 511-515 